\title{
Synchronization Over Rapidly Time-Varying Multipath Channel for CDMA Downlink RAKE Receivers in Time-Division Mode
}

\author{
Eric Simon, Laurent Ros, and Kosai Raoof
}

\begin{abstract}
In this paper, we consider a time-division codedivision multiple-access (CDMA) system operating in downlink mode in a multiuser multipath channel scenario. In order to implement RAKE reception, the delays, phases, and magnitudes of the different paths need to be estimated and the estimates updated. In the context of burst transmissions, delays do not vary during a burst, and only phases and magnitudes need to be tracked. In this paper, we investigate the issue of phase tracking over rapidly time-varying multipath channels. We propose a new version of the conventional phase loop that mitigates the interpath interference effect. The optimization is based on the concept of prefiltering. The analysis shows improved tracking performance in comparison with the standard phase recovery algorithm.
\end{abstract}

Index Terms-Code-division multiple access (CDMA), downlink, multipath channels, phase estimation.

\section{INTRODUCTION}

$\mathbf{T}$ HE conventional receiver for direct-sequence codedivision multiple-access (CDMA) multipath communications is the RAKE receiver [1]. The RAKE receiver requires knowledge of the delays, phases, and magnitudes of multipath components. In a time-division system, the channel parameters are generally first obtained during an acquisition procedure and then have to be updated during the current burst. Moreover, multipath delays can safely be assumed as constants during one burst (i.e., one time slot), and only the phases and the magnitudes of the channel coefficients have to be tracked. In this paper, we assume that the estimated path delays are perfectly known and constant, and we will focus on the decision-directed phase-tracking problem. Note that if this assumption is not valid, a more general approach exists [2]. Also, other receivers do not first estimate the channel but perform a whole strategy detection.

Manuscript received March 11, 2004; revised July 7, 2005, December 9, 2005, and June 25, 2006. This paper was presented in part at the European Signal Processing Conference (Eusipco), Vienna, Austria, September 2004. The review of this paper was coordinated by Prof. E. Sourour.

E. Simon was with the Laboratory of Image and Signal (LIS), Center National de la Recherche Scientifique (CNRS), Institut National Polytechnique de Grenoble (INPG), BP46 38402 Saint Martin d'Hères, France. He is now with the University of Lille, IEMN/TELICE, Lille, France (e-mail: eric.simon@ univ-lille1.fr).

L. Ros and K. Raoof are with the Laboratory of Image and Signal (LIS), Center National de la Recherche Scientifique (CNRS), Institut National Polytechnique de Grenoble (INPG), BP46 38402 Saint Martin d'Hères, France (e-mail: laurent.ros@lis.inpg.fr; kosai.raoof@lis.inpg.fr).

Digital Object Identifier 10.1109/TVT.2007.897664
Optimal maximum likelihood (ML) synchronizers for multiuser linear modulations in multipath context have been well known [3], [4] for many years, but due to the complexity of this estimation process, it is not suitable for practical systems. Synchronizers for frequency-selective channels are mostly taken from the additive white Gaussian noise channel, which leads to the use of a conventional single-path phase loop. According to [5], it is not so much because of their good performance but because of the lack of better solutions. For CDMA systems, the wide bandwidth of the signature requires the use of such a simple conventional algorithm in each branch of the RAKE receiver, i.e., for each multipath component. Indeed, the single-path phase-tracking algorithm performs well if paths are sufficiently spaced (typically more than one chip) and if the used signatures have quasi-perfect self- and cross-correlation functions, but in the more general case, the phase loop relative to one path may be strongly influenced by the additional paths, which results in a biased equilibrium point. In this paper, we propose an improvement to the conventional single-path phasetracking algorithm, which mitigates the adjacent path effect.

The problem is very similar in the timing recovery context with a multipath channel, where two possible methods are usually being considered. The first one consists of reconstructing the multipath interference in order to cancel it [6], [7], but the compensation term needs complete channel state information (including path magnitudes) and has to be updated each time the channel changes. The second method consists of minimizing the multipath interference [8], [9]. In [9], the estimation bias due to multipath is cancelled thanks to an adaptive timing error detector, but the estimation variance is not taken into account. In [8], the new scheme is not robust to channel phase and magnitude variations. Consequently, this scheme needs to be updated each time the channel changes, which leads to convergence problems in the case of fast time-varying channels. Now, for the phase recovery problem, our approach is similar to the second one, but improving the solution in order to make the new algorithm robust to channel coefficient variations.

Our main contribution is the inclusion of a special prefilter in the single-path tracking algorithm of each path, which is designed so that the loop operates at the desired equilibrium point (by canceling the estimation bias due to other paths), while minimizing the phase error variance. The interesting feature of this prefilter is that it does not need to be adaptive while keeping at zero the estimation bias. It is achieved by taking advantage of the nonvariation during the burst of the 
delays. Assuming perfect knowledge and separation of these delays by the channel acquisition procedure, our phase-tracking algorithm can perform correctly even if delays have a separation of less than one chip. The concept of prefiltering has been studied before by D'Andrea and Luise [10] for minimizing the timing variance for the clock recovery scheme proposed by Gardner [11] in a single-path binary phase-shift keying (PSK) scenario. Then, the authors have revisited this concept for a code-tracking loop in a multiuser CDMA system [12]. In this paper, we expand the concept to the phase recovery task but in a new perspective for bias cancellation.

After a detailed description of the system model in Section II, we introduce the standard phase loop in Section III. Its improved version including the prefilter is discussed in Section IV. Numerical results are discussed in Section V, and conclusions are given in Section VI.

\section{System Model}

The continuous-time baseband representation of the downlink-transmitted signal at the base station is modeled as

$$
x(t)=T_{s} \sum_{k=1}^{K} \sum_{n} a_{k[n]} s_{k}\left(t-n T_{s}\right)
$$

where $a_{k[n]}$ 's are the uncorrelated quaternary PSK symbols with power $A^{2}$, which is transmitted by the $k$ th source at time $n T s . K$ is the number of users. $s_{k}(t)$ is the signature of the $k$ th user, which results from the convolution between the $k$ th spreading code $\left\{c_{k}\right\}$ and the half-Nyquist filter $h_{e}$ (square root raised cosine filter), i.e.,

$$
s_{k}(\tau)=\sum_{q=0}^{Q-1} c_{k[q]} h_{e}\left(\tau-q T_{c}\right)
$$

where $T_{c}=T_{s} / Q$ is the chip duration, $Q$ is the spreading factor, and $c_{k[q]}$ 's, $q=0, \ldots, Q-1$ are the chips.

Without loss of generality, we assume that the user of interest is user 1 . We note $\Gamma_{k j}(\tau)$ as the cross-correlation function between users $k$ and $j^{1}$ :

$$
\Gamma_{k j}(\tau)=\left(s_{k} * s_{j}^{H}\right)(\tau)
$$

where ${ }^{*}$ is the convolution product. The multiuser signal from the transmitter travels through a multipath propagation channel with $L$ independent paths. The received signal can therefore be written as

$$
r(t)=\sum_{l=1}^{L} \alpha_{l}(t) x\left(t-\tau_{l}\right)+w(t)
$$

where $\alpha_{l}(t)=\rho_{l} e^{j \theta_{l}(t)}$ is the $l$ th complex path tap, $\tau_{l}$ is the respective path delay, and $w(t)$ is an additive white complex Gaussian noise with a two-sided power spectral density

\footnotetext{
${ }^{1}$ As a convention, the exponent $(\cdot)^{H}$ represents the Hermitian transformation, i.e., $f^{H}(t)=f^{*}(-t)$, for a given function $f$ and Hermitian transposition for vectors.
}

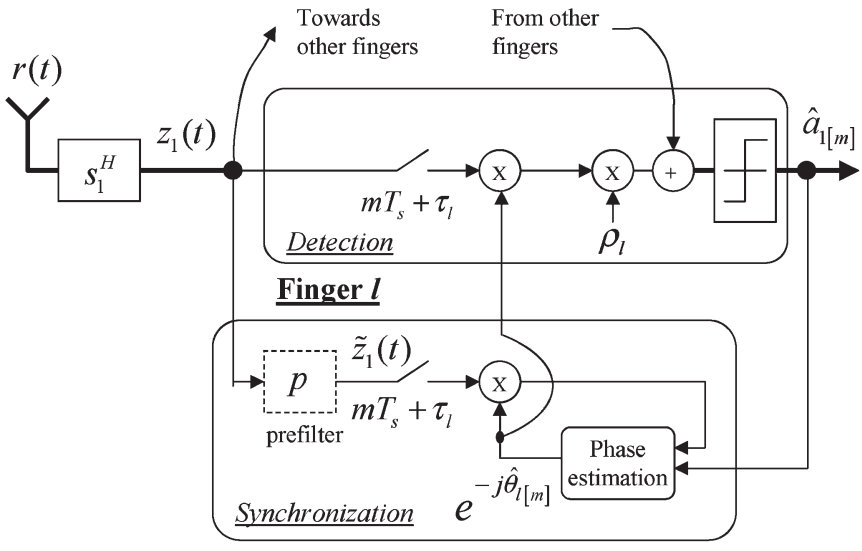

Fig. 1. Conventional phase loop (without the prefilter $p$ ) or its improved version (with the prefilter) in a RAKE receiver.

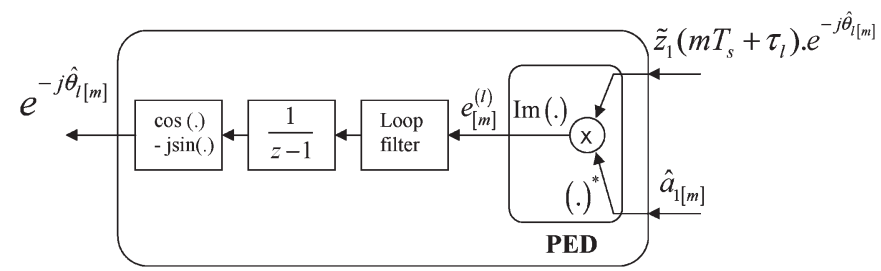

Fig. 2. Phase estimation scheme for the $l$ th path.

$2 N_{0}$. The magnitudes $\rho_{l}$ 's are supposed to be constant for the analysis, but simulations will be realized with time-variant magnitudes.

The receiver is a RAKE receiver. We note $\hat{a}_{1[m]}$ as the decision at time $m T_{s}$. The RAKE receiver needs estimates for the path delays $\tau_{l}$ and the fading coefficients $\alpha_{l}, l=1, \ldots, L$. The fading coefficients are assumed to be constant over one symbol interval. The path delays are assumed to be constant. It will be assumed that perfect timing is available, i.e., $\hat{\tau}_{l}=\tau_{l}$. In the following, the phase recovery task will be investigated. Each branch of the RAKE receiver has its own phase loop. The phase loop will be the topic of the next section.

\section{Conventional Phase Loop}

The ML estimation methods derived for a single-path singleuser channel model [13], [14] are often used in wideband CDMA to perform independent phase tracking of each desired multipath component. This simple conventional decisiondirected phase loop may be viewed as a generalization of the well-known Costas loop [14].

This phase loop is inserted in each branch of the RAKE receiver. Let us now consider the lth RAKE finger (cf. Fig. 1 without the prefilter and Fig. 2). Its purpose is to estimate the channel phase $\theta_{l}$. The estimate of $\theta_{l}$ is updated at a symbol rate by a loop error signal $e_{[m]}^{(l)}$ filtered by the digital loop filter $g_{[m]}^{(l)}$. The recursive equation of the phase loop is defined as

$$
\hat{\theta}_{l[m+1]}=\hat{\theta}_{l[m]}+\left(g^{(l)} * e^{(l)}\right)_{[m]}
$$

where $\hat{\theta}_{l[m]}$ is the estimate of $\theta_{l}$ at time $m T_{s}$. 
The loop error signal provided by the phase error detector (PED) at time $m T_{s}$ is computed as

$$
e_{[m]}^{(l)}=\operatorname{Im}\left\{e^{-j \hat{\theta}_{l[m]}} \hat{a}_{1[m]}^{*} z_{1}\left(m T_{s}+\tau_{l}\right)\right\}
$$

where $z_{1}(t)$ is the output of the matched filter $s_{1}^{H}(\tau)$ when $r(t)$ is applied, i.e., $z_{1}(t)=\left(r * s_{1}^{H}\right)(t)$. Using (1) and (2) in the above equation yields

$z_{1}(t)=T_{s} \sum_{l_{1}=1}^{L} \alpha_{l_{1}}(t) \sum_{k=1}^{K} \sum_{n} a_{k[n]} \Gamma_{k 1}\left(t-n T_{s}-\tau_{l_{1}}\right)+n(t)$

where $n(t)$ is the filtered version of $w(t)$.

In the following analysis, we will assume perfect detection, i.e., $\hat{a}_{1[\mathrm{~m}]}=a_{1[\mathrm{~m}]}$. The effect of decision errors will be considered in Section V. Substituting $z_{1}(t)$ for (4) in the above equation yields (5), shown at the bottom of the page. The error signal is composed of a useful term, together with interference terms, and a noise term. The useful term corresponds to the error signal in a single-path channel context. It gives an indication about phase error magnitude and sign. We will see in the sequel that various interference components do not have the same effect on performance. Indeed, the interpath interference (5) (i.e., interference related to the desired symbol due to the multipath combination) results in a bias on the tracked phase, whereas the multipath intersymbol interference (ISI), the multipath multiple access interference (MAI), and the noise term increase the tracked phase variance.

The loop error signal can be decomposed as the sum of a statistical expectation $\mathrm{E}\left\{e_{[m]}^{(l)} \mid \hat{\theta}_{l[m]}\right\}$ and a zero-mean disturbance $N_{[m]}^{(l)}$ called loop noise. The first term, which is called S-curve, is a function of the phase error $\theta_{l}-\hat{\theta}_{l[m]}: \mathrm{E}\left\{e_{[m]}^{(l)} \mid \hat{\theta}_{l[m]}\right\}=$ $S\left(\theta_{l}-\hat{\theta}_{l[m]}\right)$. Hence, we obtain

$$
e_{[m]}^{(l)}=S\left(\theta_{l}-\hat{\theta}_{l[m]}\right)+N_{[m]}^{(l)} .
$$

We note $\epsilon_{\theta_{l}[m]}=\theta_{l}-\hat{\theta}_{l[m]}$ as the phase error at time $m T_{s}$. Using symbol decorrelation, we compute the error signal expectation conditioned on fixed values of phase estimation $\left(\hat{\theta}_{l[m]}=\hat{\theta}_{l}\right)$, which yields the S-curve expression for the $l$ th finger (see Appendix A):

$$
\begin{aligned}
S^{(l)}\left(\epsilon_{\theta_{l}}\right) & =T_{s} A^{2} \rho_{l} \Gamma_{11}(0) \sin \left(\epsilon_{\theta_{l}}\right) \\
+ & \underbrace{\operatorname{Im}\left\{T_{s} A^{2} \sum_{l_{1} \neq l} \rho_{l_{1}} e^{j\left(\epsilon_{\theta_{l}}+\theta_{l_{1}}-\theta_{l}\right)} \Gamma_{11}\left(\tau_{l}-\tau_{l_{1}}\right)\right\}}_{\text {bias }} .
\end{aligned}
$$

Stable equilibrium points are determined by the positive-going zero crossings of $S^{(l)}\left(\epsilon_{\theta_{l}}\right)$ [14]. If we assume a single-path channel $(L=1)$, the S-curve only consists of the first term of (6). Note that $\sin (0)=0$, so that $\epsilon_{\theta_{l}}=0$ is a stable equilibrium point, which yields unbiased phase estimation. So as expected, the conventional PED operates correctly in the case of a singlepath channel or in the case of ideal self-correlation function such that $\Gamma_{11}(\tau)=0$ for $\tau \neq 0$. Otherwise, an additional term (6) depending on adjacent paths influences the PED output. Hence, depending on the delays and coefficients of other paths, the PED output appears to be biased.

In the context of small phase error fluctuations, it is possible to linearize the S-curve around its stable equilibrium point $\epsilon_{\theta_{l} \text { eq }}[14]$ as

$$
S^{(l)}\left(\epsilon_{\theta_{l}}\right)=D^{(l)} \cdot \epsilon_{\theta_{l}}-D^{(l)} \cdot \epsilon_{\theta_{l} \mathrm{eq}}
$$

where $D^{(l)}=\left.\left(\partial S^{(l)}\left(\epsilon_{\theta_{l}}\right) / \partial \epsilon_{\theta_{l}}\right)\right|_{\epsilon_{\theta_{l}}=\epsilon_{\theta_{l} \text { eq }}}$ is the S-curve slope at this point.

The corresponding tracking performance is given by the tracked phase variance. The phase variance computation with the linearized model described above gives [14]

$$
\sigma_{\theta_{l}}^{2} \approx 2 B_{P} T_{s} \frac{\Gamma_{N^{(l)}[0]}}{D^{(l)^{2}}}
$$

where $\Gamma_{N^{(l)}}$ is the loop noise autocorrelation $N^{(l)}$, and $B_{P}$ is the loop bandwidth. Multipaths not only affect the PED output on average as seen above but also considerably degrade system tracking performance. Phase variance is strongly affected by multiuser interference and by adjacent paths. In the following,

$$
\begin{aligned}
& e_{[m]}^{(l)}=\underbrace{T_{s} A^{2} \rho_{l} \Gamma_{11}(0) \sin \left(\epsilon_{\theta_{l[m]}}\right)}_{\text {useful }}+\underbrace{T_{s} \operatorname{Im}\left\{e^{\left.-j \hat{\theta}_{l[m]} \hat{a}_{1[m]}^{*} \sum_{l_{1} \neq l} \alpha_{l_{1}} a_{1[m]} \Gamma_{11}\left(\hat{\tau}_{l}-\tau_{l_{1}}\right)\right\}}\right.}_{\text {interpath interference }} \\
& +\underbrace{T_{s} \operatorname{Im}\left\{e^{-j \hat{\theta}_{l[m]}} \hat{a}_{1[m]}^{*} \sum_{l_{1}=1}^{L} \alpha_{l_{1}} \sum_{n \neq m} a_{1[n]} \Gamma_{11}\left((m-n) T_{s}+\hat{\tau}_{l}-\tau_{l_{1}}\right)\right\}}_{\text {multipath ISI }} \\
& +\underbrace{T_{s} \operatorname{Im}\left\{e^{-j \hat{\theta}_{l[m]}} \hat{a}_{1[m]}^{*} \sum_{l_{1}=1}^{L} \alpha_{l_{1}} \sum_{k=2}^{K} \sum_{n} a_{k[n]} \Gamma_{k 1}\left((m-n) T_{s}+\hat{\tau}_{l}-\tau_{l_{1}}\right)\right\}}_{\text {multipath MAI }}+\underbrace{T_{s} \operatorname{Im}\left\{e^{\left.-j \hat{\theta}_{l[m]} \hat{a}_{1[m]}^{*} n_{1}\left(m T_{s}+\hat{\tau}_{l}\right)\right\}}\right.}_{\text {noise term }}
\end{aligned}
$$


we will investigate improved versions of this conventional phase loop including multipath compensation.

\section{ImProvement of the Phase LoOP}

We propose in this section a new version of the PED, which is better suited for a multiuser system with a multipath channel. For each RAKE finger, we insert a prefilter of finite impulse response $p_{[i]}, i=-N, \ldots, N$, in the synchronization scheme, as illustrated in Fig. 1. The prefilter works at a rate of two samples per chip at input. The following notations will be used in this section:

$$
\begin{gathered}
\underline{p}=\left[p_{[-N]}, \ldots, p_{[N]}\right]^{T} \\
\tilde{f}(t)=\sum_{i=-N}^{N} p_{[i]} f\left(t-i \frac{T_{c}}{2}\right)
\end{gathered}
$$

where $f$ represents any desired function (i.e., $\tilde{f}$ is the prefiltered version of $f$ ).

The error signal (3) now becomes

$$
e_{[m]}^{(l)}=\operatorname{Im}\left\{e^{-j \hat{\theta}_{l[m]}} \hat{a}_{1[m]}^{*} \tilde{z}_{1}\left(m T_{s}+\tau_{l}\right)\right\}
$$

where $\tilde{z}_{1}(t)$ is obtained by using (4) in (8) as

$\tilde{z}_{1}(t)=T_{s} \sum_{l_{1}=1}^{L} \alpha_{l_{1}}(t) \sum_{k=1}^{K} \sum_{n} a_{k[n]} \tilde{\Gamma}_{k 1}\left(t-n T_{s}-\tau_{l_{1}}\right)+\tilde{n}(t)$.

A criterion for calculating the prefilter coefficients is being discussed. Two approaches are being considered. The first one is a multiuser approach that assumes that information of all $K$ active codes is available. The second one is a single-user approach that assumes knowledge of the desired code only (code 1 by convention).

\section{A. Multiuser Bias Cancelation-Phases and Magnitudes Independent (MBC-PMI) Prefilter}

The prefilter is designed for canceling bias due to adjacent paths (i.e., for shifting the stable equilibrium point $\epsilon_{\theta_{l} \text { eq }}$ at the origin) and for minimizing phase variance. With the prefilter, the expression of the S-curve becomes (see Appendix A)

$$
S^{(l)}\left(\epsilon_{\theta_{l}}\right)=\operatorname{Im}\left\{T_{s} A^{2} \sum_{l_{1}=1}^{L} \rho_{l_{1}} e^{j\left(\epsilon_{\theta_{l}}+\theta_{l_{1}}-\theta_{l}\right)} \tilde{\Gamma}_{11}\left(\tau_{l}-\tau_{l_{1}}\right)\right\} .
$$

The first idea for canceling the bias would be to choose the constraint

$$
S^{(l)}(0)=0 .
$$

However, the S-curve depends on the path phases. Indeed, the optimum prefilter coefficients would be computed given a set of initial phases, and the bias would not be maintained to zero for any change in channel coefficients. Hence, in the case of fast variation of the phases, the obtained prefilter would not be suitable any more. Then, it would be necessary to update the prefilter coefficients, which may lead to convergence problems under rapidly time-varying channel. In order to avoid prefilter updating, we suggest modifying the foregoing constraint. The modification consists of making the new optimum prefilter independent of the phase values.

The purpose of the prefilter is now to correct the correlation shape $\Gamma_{11}$ in (6) to force it to zero at the locations of adjacent paths:

$$
\begin{aligned}
& \operatorname{Re}\left\{\tilde{\Gamma}_{11}\left(\tau_{l}-\tau_{l_{1}}\right)\right\}=0, \quad l_{1}=1, \ldots, L, \quad l_{1} \neq l \\
& \operatorname{Im}\left\{\tilde{\Gamma}_{11}\left(\tau_{l}-\tau_{l_{1}}\right)\right\}=0, \quad l_{1}=1, \ldots, L, \quad l_{1} \neq l .
\end{aligned}
$$

Thus, we make the S-curve independent of the unwanted multipaths. With the additional constraint

$$
\operatorname{Im}\left\{\tilde{\Gamma}_{11}(0)\right\}=0
$$

the bias is maintained to zero even if the path complex amplitudes have strong variation as long as the delays are time invariant. To avoid the zero solution, we add another constraint that normalizes the S-curve slope at $\epsilon_{\theta_{l}}=0$. Since the unbiased $\mathrm{S}$-curve does not depend on the adjacent paths any more, this constraint on the S-curve slope is

$$
T_{s} A^{2} \rho_{l} \operatorname{Re}\left\{\tilde{\Gamma}_{11}(0)\right\}=1 .
$$

On the other hand, the phase variance is also a function of the channel coefficient phases. The phase variance computation as a function of prefilter coefficients is given in Appendix B. We choose to minimize only the term of the variance that does not depend on the phase values. The new function to be minimized is thus given by

$$
\phi(\underline{p})=\phi_{\mathrm{ISI}}(\underline{p})+\phi_{\mathrm{MAI}}(\underline{p})+\Gamma_{\mathrm{TN}}(\underline{p})
$$

where

$$
\begin{aligned}
\phi_{\mathrm{ISI}}(\underline{p}) & =\frac{1}{2} T_{s}^{2} A^{4} \sum_{l_{1}=1}^{L} \rho_{l_{1}}^{2} \sum_{n_{1} \neq 0}\left|\tilde{\Gamma}_{11}\left(n_{1} T_{s}+\hat{\tau}_{l}-\tau_{l_{1}}\right)\right|^{2} \\
\phi_{\mathrm{MAI}}(\underline{p}) & =\frac{1}{2} T_{s}^{2} A^{4} \sum_{l_{1}=1}^{L} \rho_{l_{1}}^{2} \sum_{k=2}^{K} \sum_{n_{1}}\left|\tilde{\Gamma}_{k 1}\left(n_{1} T_{s}+\hat{\tau}_{l}-\tau_{l_{1}}\right)\right|^{2} .
\end{aligned}
$$

The additive noise term $\Gamma_{\mathrm{TN}}(\underline{p})$ is given by

$\Gamma_{\mathrm{TN}}(\underline{p})=\frac{1}{2} \operatorname{Re}\left\{2 A^{2} N_{0} \sum_{m=-N}^{N} \sum_{q=-N}^{N} \Gamma_{11}\left((m-q) \frac{T_{c}}{2}\right) p_{[m]} p_{[q]}^{*}\right\}$.

It is now possible to sum up the criterion that is not conditioned on the phase values any more as follows:

- constrained minimization of $\phi(\underline{p})$;

- zero crossing of S-curve at $\epsilon_{\theta_{l}}=0$ [(9) and (10)];

- slope of S-curve normalized at $\epsilon_{\theta_{l}}=0$ (11). 
We propose now to use matrix notations to carry out the constrained minimization. Let us define the $(2 N+1) \times 1$ following vector:

$$
\underline{u}_{k, n}^{l, l_{1}}=\left[\begin{array}{c}
\Gamma_{k 1}\left(n T_{s}+N \frac{T_{c}}{2}+\tau_{l}-\tau_{l_{1}}\right) \\
\vdots \\
\Gamma_{k 1}\left(n T_{s}-N \frac{T_{c}}{2}+\tau_{l}-\tau_{l_{1}}\right)
\end{array}\right] .
$$

The function to be minimized $\phi(\underline{p})$ given in (12) can be written as a quadratic form

$$
\phi(\underline{p})=\underline{p}^{H} \underline{\underline{\phi}} \underline{p}
$$

where $\phi=\phi_{\mathrm{ISI}}+\underline{\underline{\mathrm{MAI}}}+\underline{\Gamma_{\mathrm{TN}}}$ is the matrix containing the three disturbance terms, i.e., ISIs, MAIs, and thermal noise perturbation. $\phi_{\mathrm{ISI}}$ and $\underline{\underline{\mathrm{MAI}}}$ are defined as

$$
\begin{aligned}
\underline{\underline{\phi_{\mathrm{ISI}}}} & =\frac{1}{2} T_{s}^{2} A^{4} \sum_{l_{1}=1}^{L} \rho_{l_{1}}^{2} \sum_{n \neq 0} \underline{u}_{1, n}^{l, l_{1}} \underline{u}_{1, n}^{l, l_{1} H} \\
\underline{\underline{\phi_{\mathrm{MAI}}}} & =\frac{1}{2} T_{s}^{2} A^{4} \sum_{l_{1}=1}^{L} \rho_{l_{1}}^{2} \sum_{k=2}^{K} \sum_{n} \underline{u}_{k, n}^{l, l_{1}} \underline{u}_{k, n}^{l, l_{1} H} .
\end{aligned}
$$

Now let us consider the $(2 N+1) \times(2 N+1)$ matrix $\underline{\underline{B}}=$ $\left[b_{m q}\right]$ with

$$
b_{m q}=\Gamma_{11}\left((m-q) \frac{T_{c}}{2}\right) .
$$

The expression of $\underline{\underline{\Gamma_{\mathrm{TN}}}}$ is given by

$$
\underline{\underline{\Gamma_{\mathrm{TN}}}}=A^{2} N_{0} \underline{\underline{B}}
$$

For notation simplicity and without loss of generality, the desired path will be the first path (i.e., $l=1$ ) from now on. Let $\underline{C}$ be the following $2 L \times 1$ vector:

$$
\begin{gathered}
\underline{C}=\left[\left(\operatorname{Re}\left\{\underline{p}^{T} \underline{u}_{1,0}^{1,1}\right\}-\frac{1}{\rho_{1} T_{s} A^{2}}\right), \operatorname{Re}\left\{\underline{p}^{T} \underline{u}_{1,0}^{1,2}\right\}, \ldots,\right. \\
\left.\operatorname{Re}\left\{\underline{p}^{T} \underline{u}_{1,0}^{1, L}\right\}, \operatorname{Im}\left\{\underline{p}^{T} \underline{u}_{1,0}^{1,1}\right\}, \ldots, \operatorname{Im}\left\{\underline{p}^{T} \underline{u}_{1,0}^{1, L}\right\}\right]^{T} .
\end{gathered}
$$

The constraints may be expressed as

$$
\underline{C}=\underline{0}
$$

where $\underline{0}$ is the null vector. This problem of minimization under constraints may be solved with the Lagrange multiplier method [15]. We form the Lagrange combination

$$
F(\underline{p}, \underline{\lambda})=\frac{1}{2} \underline{p}^{H} \underline{\underline{\phi}} \underline{p}+\underline{C}^{T} \underline{\lambda}
$$

where $\underline{\lambda}$ is a $2 L \times 1$ vector containing the Lagrange multipliers. We compute the complex gradient of $F$ with respect to $\underline{p}$, and we find the values of $p$ that make the gradient zero:

$$
\underline{\nabla}_{p} F(\underline{p}, \underline{\lambda})=\underline{\underline{\phi}} \underline{p}+\underline{\underline{G}} \underline{\lambda}=0
$$

where $\underline{\underline{G}}$ is a $(2 N+1) \times 2 L$ matrix defined by

$$
\underline{\underline{G}}=\left[\underline{u}_{1,0}^{1,1^{*}}, \ldots, \underline{u}_{1,0}^{1, L^{*}}, j \underline{u}_{1,0}^{1,1^{*}}, \ldots, j \underline{u}_{1,0}^{1, L^{*}}\right] .
$$

Substituting $\underline{p}$ for $\left(-\underline{\underline{\phi}}^{-1} \underline{\underline{G}} \underline{\lambda}\right)$ in (13) yields the expression of $\underline{\lambda}$. We finally substitute this expression in (14), and we obtain

$$
\underline{p}_{o p t}=\frac{1}{T_{s} A^{2} \rho_{1}} \underline{\underline{\phi}}^{-1} \underline{\underline{G}} \underline{\underline{M}}^{-1} \underline{1}
$$

where $\underline{1}^{T}=[1,0 \ldots, 0]$, and

$$
\underline{\underline{M}}=\left[\begin{array}{c}
\operatorname{Re}\left\{\underline{u}_{1,0}^{1,1} \underline{\underline{\phi}}^{-1} \underline{\underline{G}}\right\} \\
\vdots \\
\operatorname{Re}\left\{\underline{u}_{1,0}^{1, L} \underline{\underline{\phi}}^{-1} \underline{\underline{G}}\right\} \\
\operatorname{Im}\left\{\underline{u}_{1,0}^{1,1} \underline{\underline{\phi}}^{-1} \underline{\underline{G}}\right\} \\
\vdots \\
\operatorname{Im}\left\{\underline{u}_{1,0}^{\left.1, \underline{\underline{\phi}} \underline{\underline{\phi}}^{-1} \underline{\underline{G}}\right\}}\right.
\end{array}\right] .
$$

\section{B. Single-User Bias Cancelation-PMI (SBC-PMI) Prefilter}

As the conventional RAKE receiver uses only the information relating to the desired code, it is legitimate to consider that in this context the information of all $K$ active codes is not available. This single-user approach is the topic of this section. The function to be minimized thus becomes

$$
\phi(\underline{p})=\phi_{\mathrm{ISI}}(\underline{p})+\Gamma_{\mathrm{TN}}(\underline{p}) .
$$

Then, the prefilter coefficient calculation is similar, and the solution is given by (15), where $\underline{\underline{\phi}}$ is now defined by $\underline{\underline{\phi}}=\underline{\underline{\phi_{\text {ISI }}}}+$

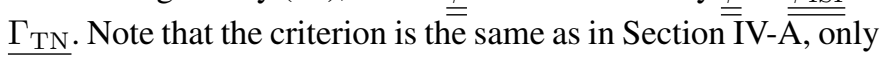
the available information is different [(16) instead of (12)]. Note also that the SBC-PMI prefilter is not well appropriate for a fully loaded system because of the high level of MAI.

\section{Numerical Results}

In this section, we present a numerical analysis of the results obtained in the previous section. A CDMA system was implemented using the parameters from Universal Mobile Telecommunications System time-division multiplexing systems. We use Hadamard codes of length $Q=16$ with the scrambling sequence $\{-1,+1,-1,-1,-1,+1,-1,-1,+1$, $-1,+1,+1,-1,+1,-1,-1\}$. The chip shaping filter is a square-root-raised cosine with roll-off factor of 0.22 . The symbol rate is $1 / T_{s}=240 \mathrm{Ksymb} / \mathrm{s}$. The loop filter impulse response $g^{(l)}$ is chosen in order to obtain a second-order loop

$$
g^{(l)}(z)=\beta_{1}+\frac{\beta_{1} \beta_{2}}{1-z^{-1}}
$$

where $\beta_{1}$ and $\beta_{2}$ are the loop filter coefficients. Relationships between the loop filter coefficients and the damping factor $\zeta$ 

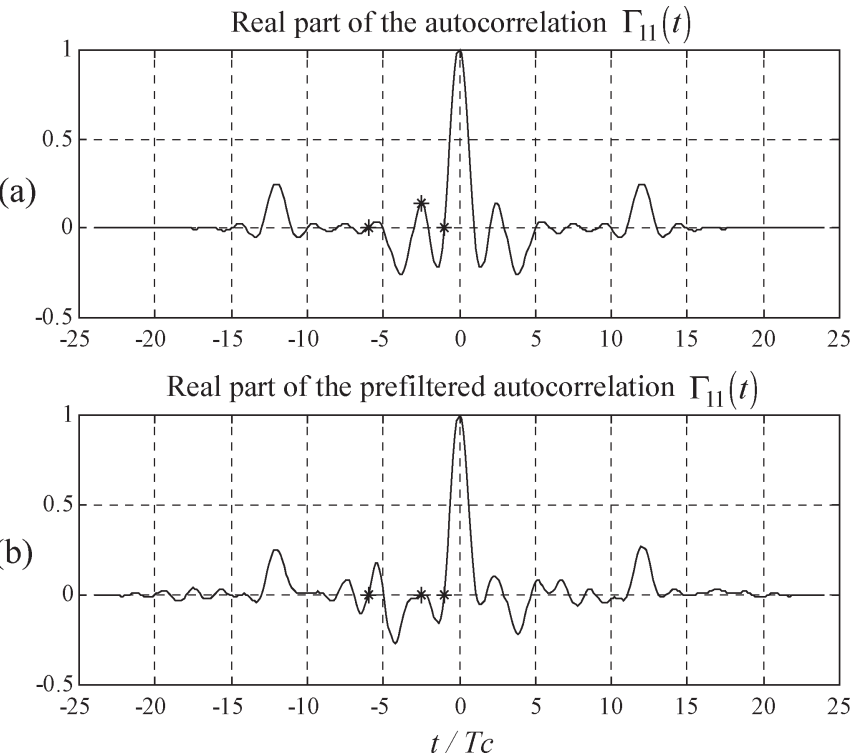

Fig. 3. (a) Correlation $\Gamma_{11}$ before prefiltering. (b) Prefiltered correlation $\tilde{\Gamma}_{11}-11$-coefficient MBC-PMI prefilter.

and the natural frequency $f_{n}$ of the loop are given by [14]

$$
\begin{aligned}
f_{n} & =\frac{\sqrt{\beta_{1} D^{(l)} \beta_{2}}}{2 \pi T_{s}} \\
\zeta & =\frac{\left(1+\beta_{2}\right) \sqrt{\beta_{1} D^{(l)}}}{2 \sqrt{\beta_{2}}} .
\end{aligned}
$$

The corresponding loop bandwidth is [14]

$$
B_{P} T_{s}=\frac{2 \beta_{2}+\beta_{1} D^{(l)}\left(2+\beta_{2}\right)}{2\left(4-\beta_{1} D^{(l)}\left(2+\beta_{2}\right)\right)} .
$$

We choose a damping factor $\zeta=0.7$ and a natural frequency $f_{n}=500 \mathrm{~Hz}$ for each loop, which yield $B_{P} T_{s}=0.007$. We consider a downlink communication with $K=5$ users. Here, four paths have been assumed at relative delays of $0 T_{c}, 2.5 T_{c}$, and $6 T_{c}$ with powers of $0,-0.9,-4.9$, and $-8 \mathrm{~dB}$. The first subsection is concerned with prefilter design and theoretical performance. As the formula of the variance (7) is obtained for a static channel, we assume in this part that the channel does not vary. The second subsection gives simulation results obtained with a time-varying channel.

\section{A. Prefilter Design and Static Performance}

Fig. 3 illustrates the effect of the MBC-PMI prefilter on the real part of the normalized correlation $\Gamma_{11}$. The prefilter is an 11-coefficient prefilter $\left(N_{p}=2 N+1=11\right)$. The current path is the first path $(l=1)$. The prefilter forces to zero the correlation at $\tau_{1}-\tau_{2}, \tau_{1}-\tau_{3}$, and $\tau_{1}-\tau_{4}$ (located by a ' $*$ ' on the figure). Hence, the resulting S-curve will not be distorted by the additional paths.

Fig. 4 shows the corresponding optimized PED S-curve compared with the conventional PED S-curve. In the latter case, it is noted that the S-curve zero crossing is not located at $0\left(\theta_{1}-\theta_{1_{\mathrm{eq}}} \approx-0.4 \mathrm{rad}\right)$. It results in a bias on the tracked phase due to the presence of adjacent paths. Fig. 4(b) shows

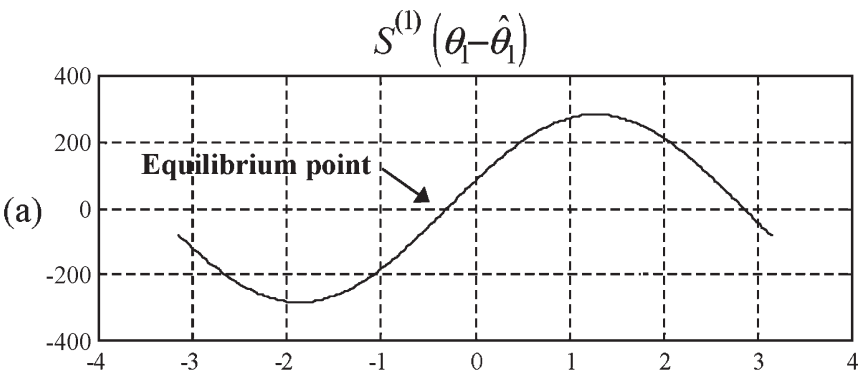

(b)

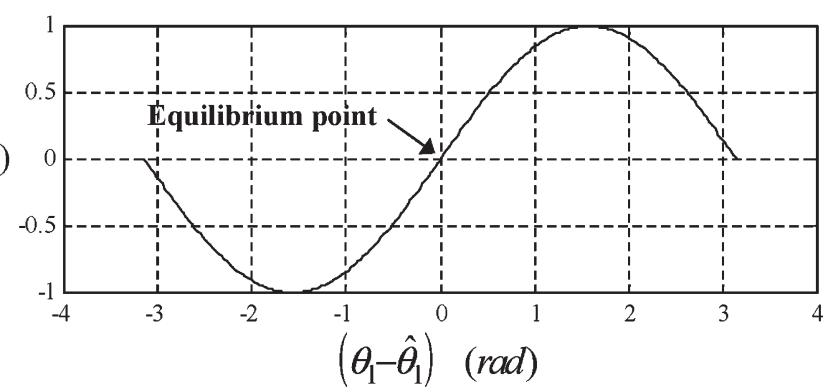

Fig. 4. (a) Conventional PED S-curve. (b) Improved PED S-curve11-coefficient MBC-PMI prefilter.

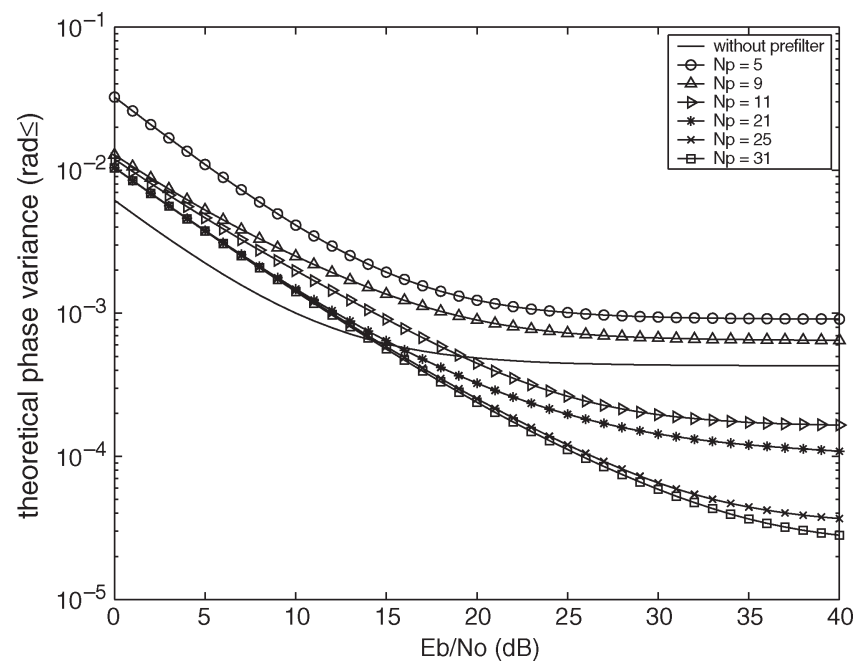

Fig. 5. Theoretical phase variance-MBC-PMI prefilter, $B_{P} T_{s}=0.007$.

the optimized PED S-curve. The zero crossing is shifted at the origin, which yields an unbiased estimation.

In the following, the theoretical phase estimation variance will be investigated. We recall that the linearized tracking performance [(7) with $\Gamma_{N^{(l)}[0]}$ given by (26)] is computed under the assumption that correct decisions are available i.e., $\hat{a}_{1[m]}=a_{1[m]}$. The effect of decision errors will be considered in the sequel by means of simulations.

To begin with, let us consider the MBC-PMI prefilter. Fig. 5 shows the phase variance as a function of $E_{b} / N_{0}$ for different values of $N_{p}$ for the first path. We also calculate the conventional loop phase variance (without prefiltering). It is shown that the conventional loop has a floor due to the multipath ISI and MAI terms. The prefilter contributes to mitigate the interference terms, and as a result, the performance curve of the phase loop with prefilter is improved. The phase variance decreases with an increasing number of prefilter coefficients. But if the 


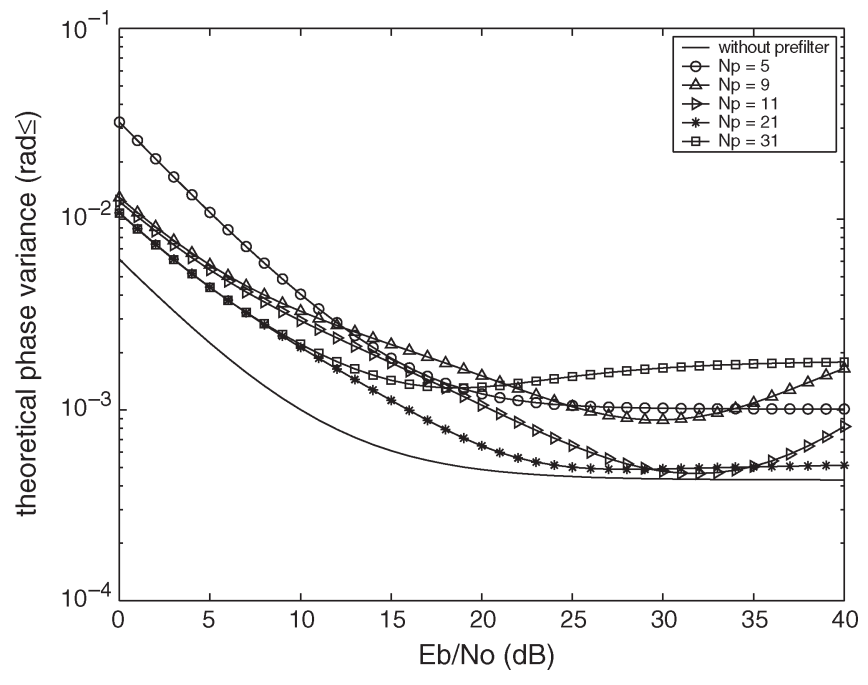

Fig. 6. Theoretical phase variance-SBC-PMI prefilter, $B_{P} T_{s}=0.007$.

number of coefficients increases beyond a certain value, we obtain a negligible improvement in performance $\left(N_{p}=31\right.$ in comparison with $\left.N_{p}=25\right)$. Hence, the choice of the coefficient number would represent a compromise between cost and performance. Regarding such a tradeoff, the best choice for the prefilter length would depend on the signal-to-noise ratio. Indeed, at high $E_{b} / N_{0}$, a large number of coefficients would have a larger impact on performance than at low $E_{b} / N_{0}$.

Fig. 6 shows the phase variance with the SBC-PMI prefilter. As explained in Section IV-B, the SBC-PMI prefilter minimizes the noise and ISI terms without taking into account the presence of other users. Consequently, it results in performance degradation in comparison with the results obtained with the MBC-PMI prefilter. Note that for high $E_{b} / N_{0}$, prefilter length has no impact on performance improvement. Indeed, for high $E_{b} / N_{0}$, performance degradation stems from the MAI, whereas the prefilter is not designed to treat the MAI.

In order to study the effect of decision errors, simulations have been carried out with MBC-PMI and SBC-PMI prefilters. The number of coefficients is $N_{p}=11$. Fig. 7 shows the curves of the phase variance obtained by simulation (solid lines) and the curves of the theoretical phase variance obtained with (7) (dashed lines). The phase variance approximation ignores the effect of decision errors so that (7) underestimates the tracking variance at low $E_{b} / N_{0}$. The difference between both curves is caused only by the additive noise and the interference terms affecting the receiver decisions.

\section{B. Simulated Tracking Performance}

The mobile speed is $v_{m}=120 \mathrm{~km} / \mathrm{h}$, i.e., $\Delta f_{d}=220 \mathrm{~Hz}$ for a carrier radio frequency of $2 \mathrm{GHz}$. With the chosen parameters $f_{n}$ and $\zeta$, the loops are suitable for a typical Jakes' model random Doppler of $220 \mathrm{~Hz}$, but the simulations are realized with a deterministic Doppler to make the analysis of the tracking easier.

The channel phase variation model is given by $\theta_{l}(t)=$ $\Delta \theta^{(l)} \sin \left(2 \pi f_{g}^{(l)} t\right)$. In the vicinity of zero crossings, the phase variation model corresponds to a deterministic Doppler model

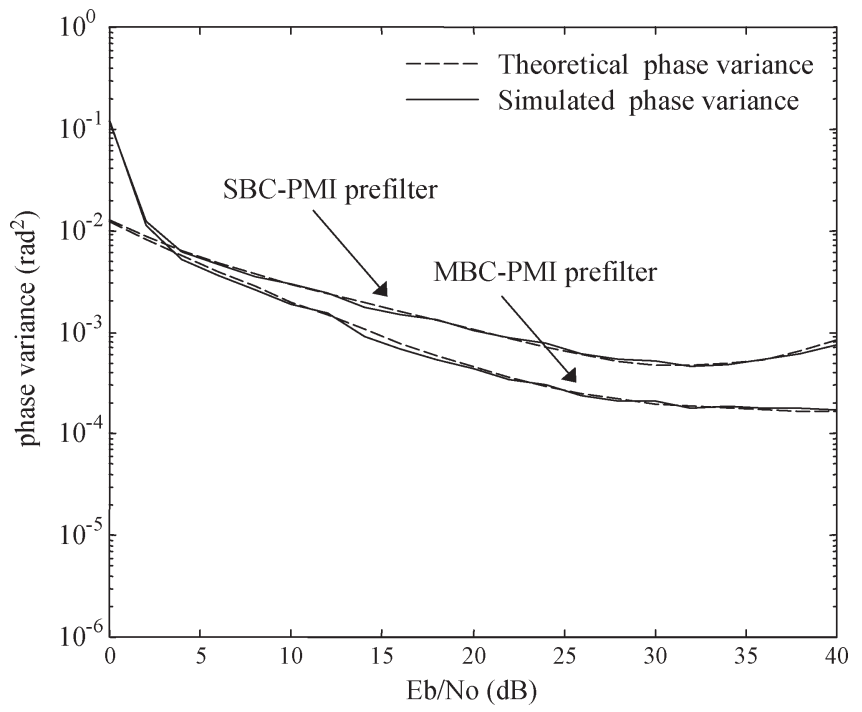

Fig. 7. Simulated phase variances $-N_{p}=11, B_{P} T_{s}=0.007$.

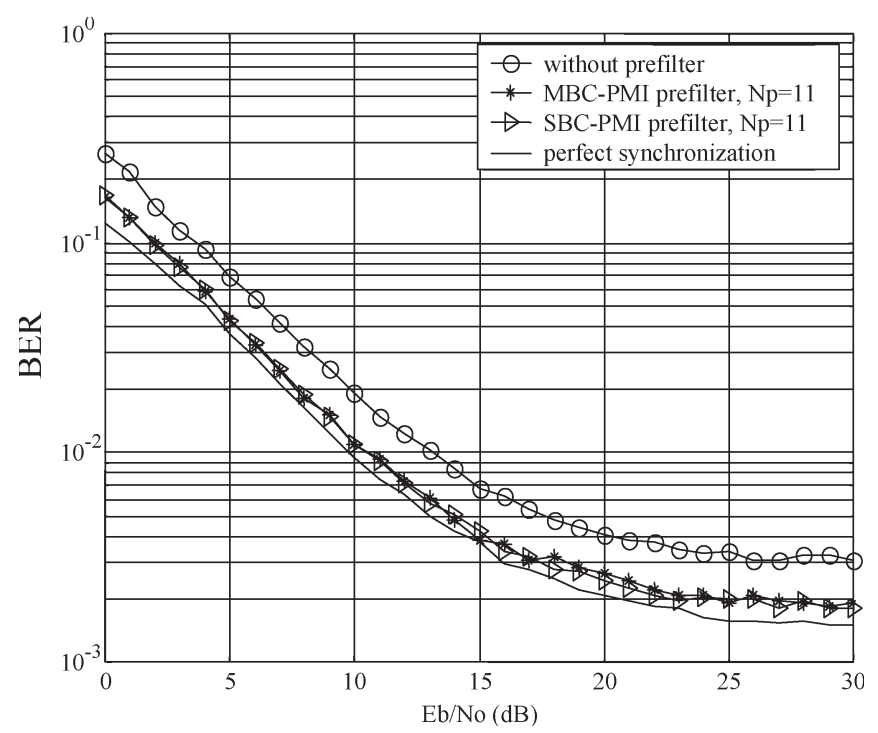

Fig. 8. BER of RAKE receiver, $v_{m}=120 \mathrm{~km} / \mathrm{h}, B_{P} T_{s}=0.007$.

with a Doppler spread $\Delta f_{d}^{(l)}=\Delta \theta^{(l)} f_{g}^{(l)}$. We choose $f_{g}^{(1)}=$ $-220 \mathrm{~Hz}, f_{g}^{(2)}=90 \mathrm{~Hz}, f_{g}^{(3)}=-50 \mathrm{~Hz}$, and $f_{g}^{(4)}=220 \mathrm{~Hz}$, and $\Delta \theta^{(1)}=1, \Delta \theta^{(2)}=2, \Delta \theta^{(3)}=4$, and $\Delta \theta^{(4)}=1$. Hence, we obtain a very different Doppler, which may occur in radio mobile scenarios: $\Delta f_{d}^{(1)}=-220 \mathrm{~Hz}, \Delta f_{d}^{(2)}=180 \mathrm{~Hz}$, $\Delta f_{d}^{(3)}=-200 \mathrm{~Hz}$, and $\Delta f_{d}^{(4)}=220 \mathrm{~Hz}$.

Fig. 8 shows the resulting bit error rates (BERs) for a signal-to-noise ratio $E_{b} / N_{0}=20 \mathrm{~dB}$ for the RAKE receiver with both loops compared to the BER obtained with perfect synchronization. At a nominal BER of $10^{-2}$, the gain achieved by the improved loop with the SBC-PMI prefilter as compared to the conventional loop is $2.5-3 \mathrm{~dB}$. The BER curve obtained with the improved loop becomes closer to the BER curve obtained with perfect synchronization. The BER obtained with the 11-coefficient MBC-PMI prefilter is shown as well. The curves obtained with both prefilters are similar. It means that the gain obtained with the MBC-PMI prefilter, as compared to the SBC-PMI prefilter in terms of phase variance, has no impact 


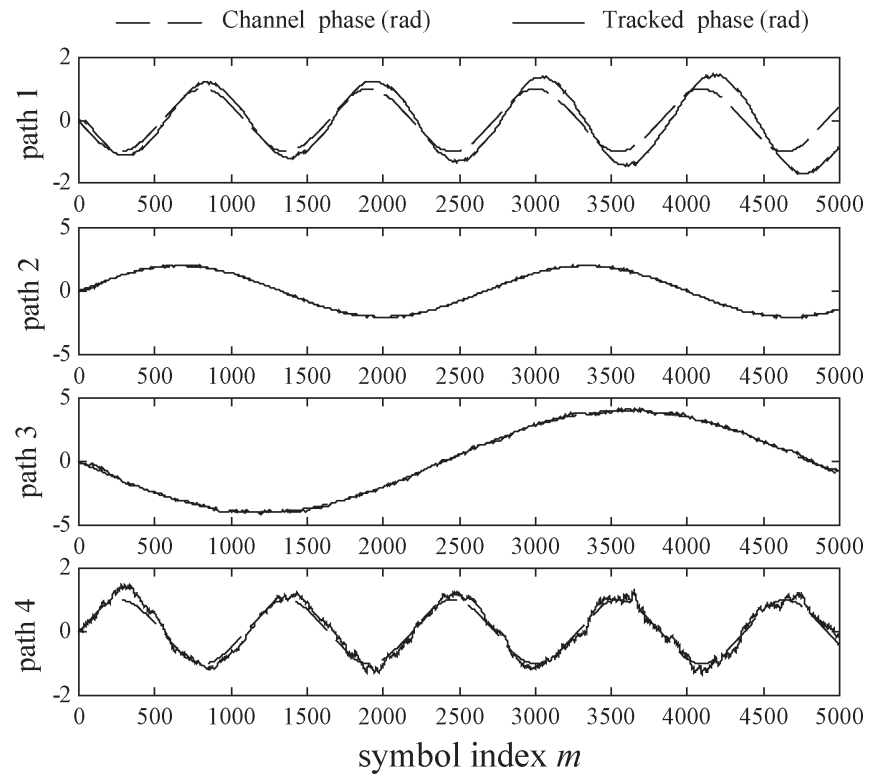

Fig. 9. Tracked phase with an 11-coefficient SBC-PMI prefilter for linear time-varying path magnitudes of $-15,10,-7$, and $5 \mathrm{~dB}$, respectively, for paths 1 to $4 . E_{b} / N_{0}=20 \mathrm{~dB}$

on the BER, but if we consider a context with more users, the improvement with the MBC-PMI prefilter is not negligible.

In the system model, time-invariant path magnitudes have been assumed. We will now discuss about the magnitude variation impact. Since the prefilter makes the bias cancellation independent of the adjacent paths, the magnitude variations have no impact on the bias cancellation. However, it is seen from (11), (17), and (26) that the magnitude variations affect the phase variance and loop parameters. Indeed, the S-curve slope $D^{(l)}(11)$ depends on the corresponding path magnitude $\rho_{l}$. Consequently, the phase variance minimization may be damaged, but above all, the loop parameters and so the tracking capabilities are modified. A first way to resolve the latest problem is adapting this phase loop parameter from a joint magnitude tracking procedure. Indeed, thanks to interpath interference cancellation, the output of the prefilter could be easily used to complete the previous algorithm in order to estimate the magnitude, as proposed in [16].

Otherwise, without tracking magnitudes, we will show by means of simulation that the prefilter is robust to magnitude variations. We use the same variation model for the phases, and we consider linear variations of the magnitude of -15 , $10,-7$, and $5 \mathrm{~dB}$ for paths 1 to 4 , respectively, from $0 T_{s}$ to $5000 T_{s}$ (see Fig. 9). Hence, for the first path, the natural frequency at the instant $5000 T_{s}$ becomes $f_{n}=210 \mathrm{~Hz}$, whereas $f_{g}^{(1)}=-220 \mathrm{~Hz}$. Consequently, as mentioned above, the loop is not well suited to follow the channel phase anymore. A way for overcoming this problem is to initially choose a higher natural frequency. Note that the magnitude variation impact on the phase variance is negligible. Moreover, note that for this scenario of magnitude variation, the first path becomes not preponderant for signal detection.

The effect of the prefilter is most obvious if spacings are noninteger multiples of the chip duration. Let us consider the following second channel: four paths have been assumed at

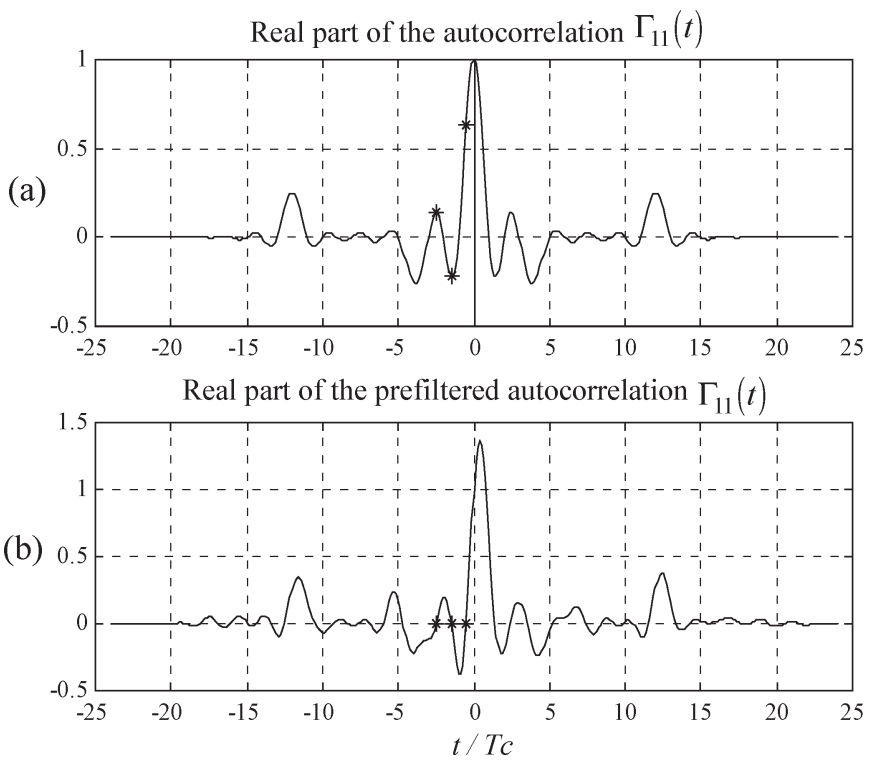

Fig. 10. (a) Correlation $\Gamma_{11}$ before prefiltering. (b) Prefiltered correlation $\tilde{\Gamma}_{11}$-11-coefficient SBC-PMI prefilter (second channel).
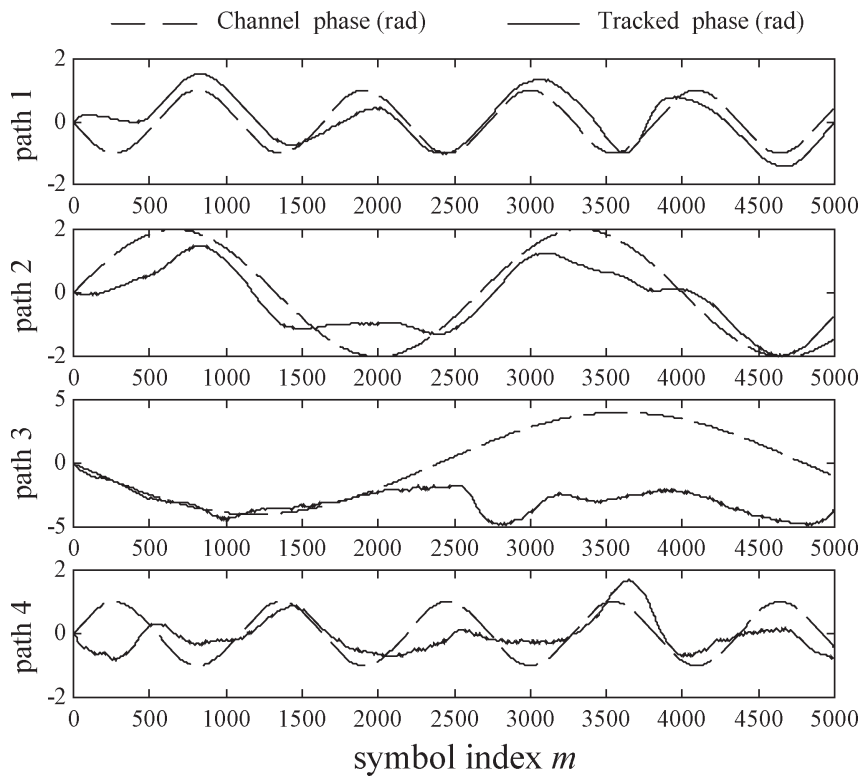

Fig. 11. Tracked phase with the conventional phase loop (second channel). $E_{b} / N_{0}=20 \mathrm{~dB}$.

relative delays of $0 T_{c}, 0.5 T_{c}, 1.5 T_{c}$, and $2.5 T_{c}$ with powers of $0,-0.9,-4.9$, and $-8 \mathrm{~dB}$. The variation model is the same as before. The prefilter is an 11-coefficient SBC-PMI prefilter. As clearly shown in Fig. 10(a), the interpath interferences are at a maximum with such a channel. In such extreme scenarios, the conventional phase recovery loop fails. As illustrated in Fig. 11, the conventional loop is not able to track channel phases, which is not the case of the improved loop (see Fig. 12) with the SBC-PMI prefilter.

\section{CONCLUSION}

In this paper, a new advanced phase-tracking technique in a downlink time-division CDMA system appropriate to rapidly 


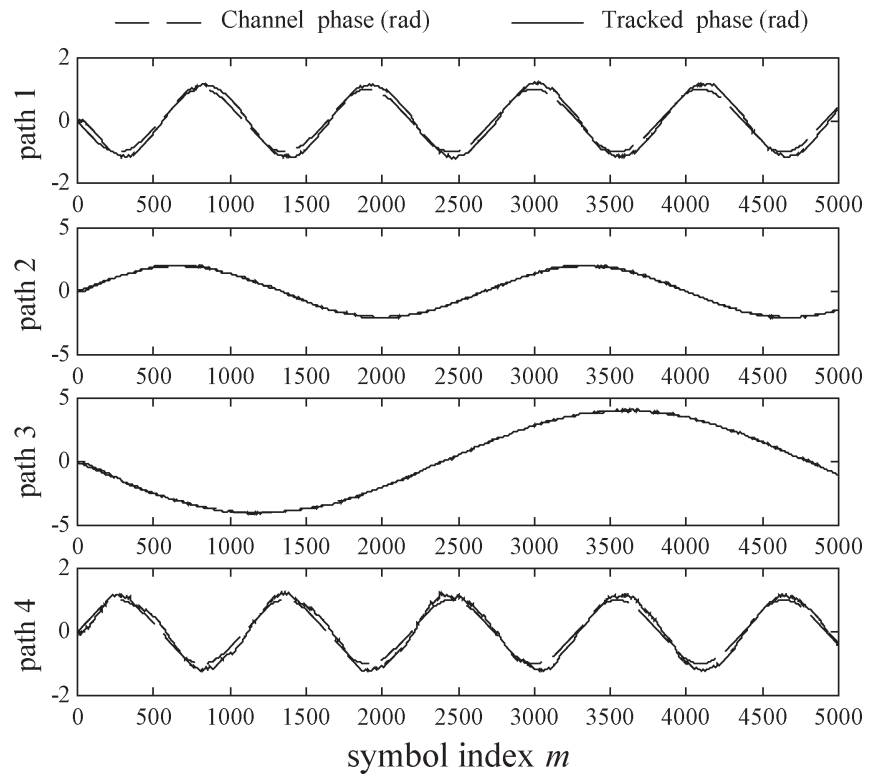

Fig. 12. Tracked phase with an 11-coefficient SBC-PMI prefilter (second channel). $E_{b} / N_{0}=20 \mathrm{~dB}$.

time-varying multipath channel is proposed. The new structure is based on a conventional single-path phase recovery loop in which a prefilter has been inserted. It is explained how to design the optimum prefilter in order to minimize the phase variance and to cancel the bias due to additional paths, independently of the channel component variations. Hence, the thus obtained prefilter does not need to be updated. For this criterion, two approaches for calculating the coefficients are being considered: the first one assumes knowledge of all $K$ active codes, and the second one assumes knowledge of the desired code only. The single-user approach corresponds to the conventional RAKE receiver context, where only the information of the desired code is used. Linear analysis and computer simulations have been employed to evaluate new tracking loop performance. Numerical results show that the new tracking loop outperforms the conventional one. Moreover, in terms of BER, the singleuser approach performs as well as the multiuser approach for a low load. Furthermore, it has been shown that in certain particular scenarios such as unresolved multipaths, the conventional phase loop fails, whereas the improved loop operates correctly.

\section{APPENDIX A \\ S-CURVE EXPRESSION}

The S-curve is the expectation of the error signal conditioned on fixed values of $\hat{\theta}_{l[m]}$ :

$$
S^{(l)}\left(\epsilon_{\theta_{l}}\right)=\mathrm{E}\left\{e_{[m]}^{(l)} \mid \hat{\theta}_{l[m]}=\hat{\theta}_{l}\right\}
$$

Substituting $e_{[m]}^{(l)}$ for (3) in the above equation yields

$$
S^{(l)}\left(\epsilon_{\theta_{l}}\right)=\mathrm{E}\left\{\operatorname{Im}\left\{e^{-j \hat{\theta}_{l}} a_{1[m]}^{*} z_{1}\left(m T_{s}+\tau_{l}\right)\right\}\right\} .
$$

Inserting (4) into (20) and computing the expected value with respect to independent identically distributed data and noise yields the $\mathrm{S}$-curve expression

$$
S^{(l)}\left(\epsilon_{\theta_{l}}\right)=\operatorname{Im}\left\{T_{s} A^{2} \sum_{l_{1}=1}^{L} \rho_{l_{1}} e^{j\left(\epsilon_{\theta_{l}}+\theta_{l_{1}}-\theta_{l}\right)} \Gamma_{11}\left(\tau_{l}-\tau_{l_{1}}\right)\right\} .
$$

The PED S-curve with the prefilter is obtained by substituting the correlation $\Gamma_{11}$ by its prefiltered version $\tilde{\Gamma}_{11}$ as

$$
S^{(l)}\left(\epsilon_{\theta_{l}}\right)=\operatorname{Im}\left\{T_{s} A^{2} \sum_{l_{1}=1}^{L} \rho_{l_{1}} e^{j\left(\epsilon_{\theta_{l}}+\theta_{l_{1}}-\theta_{l}\right)} \tilde{\Gamma}_{11}\left(\tau_{l}-\tau_{l_{1}}\right)\right\} .
$$

\section{APPENDIX B}

\section{EXPRESSiOn OF THE TRACKed Phase VARIANCE}

The purpose of this Appendix is the tracked phase variance calculation for a given prefilter. It follows from (7) that the phase variance is proportional to the loop noise autocorrelation $\Gamma_{N^{(l)}[n]}$ taken at $n=0$. The detailed computation of $\Gamma_{N^{(l)}[0]}$ is the topic of this Appendix.

The autocorrelation of $N_{[m]}^{(l)}$ is defined as

$$
\Gamma_{N^{(l)}[n]}=\mathrm{E}\left\{N_{[m]}^{(l)} N_{[m-n]}^{(l)^{*}}\right\} .
$$

Recall that the zero-mean loop noise $N_{[m]}^{(l)}$ is defined by

$$
N_{[m]}^{(l)}=e_{[m]}^{(l)}-S^{(l)}\left(\epsilon_{\theta_{l}[m]}\right) .
$$

Hence, substituting (23) in (22) yields

$$
\Gamma_{N^{(l)}[n]}=\mathrm{E}\left\{e_{[m]}^{(l)} e_{[m-n]}^{(l)^{*}}\right\}-\left|S^{(l)}\left(\epsilon_{\theta_{l}[m]}\right)\right|^{2} .
$$

The sequel is concerned with the computation of the term $\mathrm{E}\left\{e_{[m]}^{(l)} e_{[m-n]}^{(l)^{*}}\right\}$ at $n=0$. The new error signal with the prefilter can be expressed as

$$
e_{[m]}^{(l)}=\operatorname{Im}\left\{e^{-j \hat{\theta}_{l[m]}} a_{1[m]}^{*} \tilde{z}_{1}\left(m T_{s}+\tau_{l}\right)\right\} .
$$

We substitute (25) in the above expression taken at $n=0$, and we develop the product of the two imaginary parts by using the equality

$$
\operatorname{Im}\{z\} \operatorname{Im}\left\{z^{\prime}\right\}=\frac{1}{2} \operatorname{Re}\left\{z z^{\prime *}-z z^{\prime}\right\}
$$

where $z$ and $z^{\prime}$ are two complex numbers, and $z^{\prime *}$ is the complex conjugate of $z^{\prime}$.

We obtain

$$
\mathrm{E}\left\{e_{[m]}^{(l)} e_{[m]}^{(l)^{*}}\right\}=\frac{1}{2} \operatorname{Re}\left\{\mathrm{E}\left\{\psi-\psi^{\prime}\right\}\right\}
$$

where

$$
\begin{aligned}
\psi & =a_{1[m]}^{*} \tilde{z}_{1}\left(m T_{s}+\tau_{l}\right) \cdot a_{1[m]} \tilde{z}_{1}^{*}\left(m T_{s}+\tau_{l}\right) \\
\psi^{\prime} & =e^{-2 j \hat{\theta}_{l[m]}} a_{1[m]}^{*} \tilde{z}_{1}\left(m T_{s}+\tau_{l}\right) \cdot a_{1[m]}^{*} \tilde{z}_{1}\left(m T_{s}+\tau_{l}\right) .
\end{aligned}
$$


We compute the expectations $\mathrm{E}\{\psi\}$ and $\mathrm{E}\left\{\psi^{\prime}\right\}$. By using this result with (21) in (24), we obtain

$$
\Gamma_{N^{(l)}[0]}=\Gamma_{\mathrm{ISI}}+\Gamma_{\mathrm{MAI}}+\Gamma_{\mathrm{TN}}
$$

where

$$
\begin{gathered}
\Gamma_{\text {ISI }}=\frac{1}{2} \operatorname{Re}\left\{T_{s}^{2} A^{4} \sum_{l_{1}=1}^{L} \sum_{l_{2}=1}^{L} \alpha_{l_{1}} \alpha_{l_{2}}^{*} \sum_{n_{1} \neq 0} \tilde{\Gamma}_{11}\left(n_{1} T_{s}+\tau_{l}-\tau_{l_{1}}\right)\right. \\
\left.\times \tilde{\Gamma}_{11}^{*}\left(n_{1} T_{s}+\tau_{l}-\tau_{l_{2}}\right)\right\}
\end{gathered}
$$$$
\Gamma_{\mathrm{MAI}}=\frac{1}{2} \operatorname{Re}\left\{T_{s}^{2} A^{4} \sum_{l_{1}=1}^{L} \sum_{l_{2}=1}^{L} \alpha_{l_{1}} \alpha_{l_{2}}^{*}\right.
$$$$
\times \sum_{k=2}^{K} \sum_{n_{1}} \tilde{\Gamma}_{k 1}\left(n_{1} T_{s}+\tau_{l}-\tau_{l_{1}}\right)
$$$$
\left.\times \tilde{\Gamma}_{k 1}^{*}\left(n_{1} T_{s}+\tau_{l}-\tau_{l_{2}}\right)\right\}
$$$$
\Gamma_{\mathrm{TN}}=\frac{1}{2} \operatorname{Re}\left\{2 A^{2} N_{0} \sum_{m=-N}^{N} \sum_{q=-N}^{N} \Gamma_{11}\left((m-q) \frac{T_{c}}{2}\right) p_{[m]} p_{[q]}^{*}\right\} .
$$

\section{REFERENCES}

[1] G. L. Turin, "Introduction to spread-spectrum antimultipath techniques and their application to urban digital radio," Proc. IEEE, vol. 68, no. 3, pp. 328-353, Mar. 1980.

[2] A. Hansson and T. Aulin, "Generalized APP detection of continuous phase modulation over unknown ISI channels," IEEE Trans. Commun., vol. 53, no. 10, pp. 1615-1619, Oct. 2005.

[3] E. G. Ström and F. Malmsten, "A maximum likelihood approach for estimating DS-CDMA multipath fading channels," IEEE J. Sel. Areas. Commun., vol. 18, no. 1, pp. 132-140, Jan. 2000.

[4] L. Schumacher and L. Vandendorpe, "MAI mitigation in DA ML carrier phase recovery loops for DS-CDMA systems," in Proc. VTC_Fall, 1999, pp. $1850-1854$.

[5] M. Luise, U. Mengali, M. Moeneclaey, J. G. Proakis, and E. Ström, "Guest editorial signal synchronization in digital transmissions systems," IEEE J. Sel. Areas. Commun., vol. 19, no. 12, pp. 2239-2297, Dec. 2001.

[6] G. Fock, P. Schulz-Rittich, J. Baltersee, and H. Meyr, "Multipath resistant coherent timing-error-detector for DS-CDMA applications," in Proc. ISSTA, 2000, pp. 278-282.

[7] M. Guenach and L. Vandendorpe, "Tracking performance of DA and DD multiuser timing synchronizers for short code DS-CDMA systems," IEEE J. Sel. Areas. Commun., vol. 19, no. 12, pp. 2452-2461, Dec. 2001.

[8] G. Fock, P. Schulz-Rittich, and J. Baltersee, "Optimized timing-errordetector for DS-CDMA applications in multipath scenarios," in Proc. VTC-Spring, 2001, pp. 1729-1733.

[9] P. Schulz-Rittich, G. Fock, J. Baltersee, and H. Meyr, "Low complexity adaptive code tracking with improved multipath resolution for DS-CDMA communications over fading channels," in Proc. ISSSTA, 2000, pp. 30-34.

[10] A. N. D' Andrea and M. Luise, "Optimization of symbol timing recovery for QAM data demodulators," IEEE Trans. Commun., vol. 44, no. 3, pp. 399-406, Mar. 1996.
[11] F. M. Gardner, "A BPSK/QPSK timing-error detector for sampled receivers," IEEE Trans. Commun., vol. COM-34, no. 5, pp. 423-429, May 1986.

[12] E. Simon, K. Raoof, and L. Ros, "Optimization of symbol timing recovery for multi-user DS-CDMA receivers," in Proc. ICASSP, 2003, pp. 604-607.

[13] H. Meyr, M. Moeneclaey, and S. A. Fechtel, Digital Communication Receivers, ser. Wiley Series in Telecommunications and Signal Processing. Hoboken, NJ: Wiley, 1998.

[14] U. Mengali and A. N. D' Andrea, Synchronization Techniques for Digital Receivers. New York: Plenum, 1997.

[15] R. Fletcher, Practical Methods of Optimization. New York: Wiley, 1987

[16] E. Simon and L. Ros, "Adaptive multipath channel estimation in CDMA based on prefiltering and combination with a linear equalizer," in Proc. IST Mobile and Wireless Commun. Summit, Dresden, Germany, Jun. 2005.

Eric Simon received the M.S. degree in electronics engineering from École Superérieure Chimie Physique Électronique de Lyon (CPE Lyon), Lyon, France, in 1999 and the Ph.D. degree in signal processing and communications from the Institut National Polytechnique de Grenoble (INPG), Grenoble, France, in 2004.

In 2005, he was a Teaching Assistant at the INPG. The following year, he was a Post-Doctoral Researcher in the Research and Development Division, France Telecom, Rennes, France. He is currently an Associate Professor at the Institute of Electronics, Microelectronics, and Nanotechnologies (IEMN), Lille, France. His research interests are in the area of wireless communications, including synchronization problems, MIMO systems, and multicarrier transmissions.
Laurent Ros received the degree in electrical engineering from the Superior School of Electricity (Supelec), Paris, France, in 1992 and the Ph.D. degree in signal processing and communications from the Institut National Polytechnique de Grenoble (INPG), Grenoble, France, in 2001.

From 1992 to 1995, he was with the Direction of Naval Construction, Toulon, France, for one year of military service, and with France Telecom, where he worked in the area of very-low-frequency radio transmissions for submarine applications. From 1995 to 1999, he was a Research and Development Project and a Team Manager at Sodielec, Millau, France, where he worked in the design of digital modems and audio codecs for telecommunication applications. Since 1999, he has been with the "Laboratory of Image and Signal" (LIS), INPG, where he is currently an Associate Professor. His general research interests include synchronization and equalization problems, multiuser systems, and diversity techniques for wireless and digital communications.

Kosai Raoof received the degree from the College of Electrical Engineering, University of Baghdad, Baghdad, Iraq, in 1985, the M.Sc. degree in signal processing from the Institut National Polytechnique de Grenoble (INPG), Grenoble, France, in 1990, and the Ph.D. and D.H.D.R. degrees from the University of Joseph Fourier, Grenoble, in 1993 and 1998, respectively.

$\mathrm{He}$ was an Associate Professor at the University of Joseph Fourier. Since 1999, he has been with the "Laboratory of Images and Signals" (LIS), Grenoble, where his research interest is focused in the telecommunication field, especially MIMO systems, CDMA synchronization applied for 4G mobile systems, and software-defined radio. In 2004, he was a full Post-Associate Professor at the University of Joseph Fourier. He is the author of more than 50 scientific papers in the field of signal processing. Much of his research was on biomedical signal processing. 